\title{
Feasibility of spatial frequency- domain imaging for monitoring palpable breast lesions
}

Constance M. Robbins

Guruprasad Raghavan

James F. Antaki

Jana M. Kainerstorfer 


\title{
Feasibility of spatial frequency-domain imaging for monitoring palpable breast lesions
}

\author{
Constance M. Robbins, Guruprasad Raghavan, James F. Antaki, and Jana M. Kainerstorfer* \\ Carnegie Mellon University, Department of Biomedical Engineering, Pittsburgh, Pennsylvania, United States
}

\begin{abstract}
In breast cancer diagnosis and therapy monitoring, there is a need for frequent, noninvasive disease progression evaluation. Breast tumors differ from healthy tissue in mechanical stiffness as well as optical properties, which allows optical methods to detect and monitor breast lesions noninvasively. Spatial frequency-domain imaging (SFDI) is a reflectance-based diffuse optical method that can yield two-dimensional images of absolute optical properties of tissue with an inexpensive and portable system, although depth penetration is limited. Since the absorption coefficient of breast tissue is relatively low and the tissue is quite flexible, there is an opportunity for compression of tissue to bring stiff, palpable breast lesions within the detection range of SFDI. Sixteen breast tissue-mimicking phantoms were fabricated containing stiffer, more highly absorbing tumor-mimicking inclusions of varying absorption contrast and depth. These phantoms were imaged with an SFDI system at five levels of compression. An increase in absorption contrast was observed with compression, and reliable detection of each inclusion was achieved when compression was sufficient to bring the inclusion center within $\sim 12 \mathrm{~mm}$ of the phantom surface. At highest compression level, contrasts achieved with this system were comparable to those measured with single source-detector near-infrared spectroscopy. @ 2017 Society of Photo-Optical Instrumentation Engineers (SPIE) [DOI: 10.1117/1.JBO.22.12.121605]
\end{abstract}

Keywords: breast cancer; spatial frequency-domain imaging; optical mammography; compression; near-infrared light.

Paper 170354SSPR received May 31, 2017; accepted for publication Jul. 27, 2017; published online Aug. 22, 2017.

\section{Introduction}

Breast cancer is the most prevalent nonskin cancer among women. In 2014, 236,968 women were diagnosed, and 41,211 died of breast cancer. ${ }^{1}$ The most common method of screening for breast cancer is $\mathrm{x}$-ray mammography. When the results of screening mammography are suspicious, guidelines recommend breast biopsy to obtain a definitive diagnosis. ${ }^{2}$ For mammographic or sonographic findings considered "probably benign" (BI-RADS III), repeated imaging at 6 month intervals is usually recommended for nonpalpable lesions. ${ }^{3}$ However, the presence of a palpable breast lesion is often considered indication for immediate biopsy ${ }^{2,4}$ (despite evidence that palpable lesions displaying "probably benign" features have a rate of true malignancy comparable to that of nonpalpable lesions ${ }^{4-6}$ ), which indicateds the need for a noninvasive method of frequent monitoring of these lesions. Frequent monitoring of breast lesions is also of interest for patients undergoing neoadjuvant chemotherapy, ${ }^{7}$ to predict therapy outcome earlier than is possible with mammography and ultrasound. 8,9

Breast tumors are known to be highly vascularized, containing greater total hemoglobin and water concentration and lower lipid concentration than the surrounding tissue. ${ }^{10}$ Malignant breast tumors are also known to be stiffer than surrounding healthy breast tissue and often are found to be stiffer than benign abnormalities such as fibroadenoma. ${ }^{11,12}$ A variety of approaches exist to detect and monitor breast lesions based on these properties.

Optical imaging of breast tissue lends itself for monitoring palpable lesions noninvasively, as optical methods do not require exogenous contrast agents or ionizing radiation, making

*Address all correspondence to: Jana M. Kainerstorfer, E-mail: jkainers@ andrew.cmu.edu these techniques appropriate for repeated measurements. In addition, advantages of using optical methods include low cost and portability. In particular, diffuse optical imaging with nearinfrared light to measure absorption and scattering properties in breast tissue has previously been used to differentiate between healthy tissue and breast lesions, ${ }^{10,13}$ and to monitor breast cancer in patients undergoing neoadjuvant chemotherapy.,10

Among optical imaging modalities, one can distinguish between continuous wave $(\mathrm{CW})$ diffuse optical imaging systems and time- or frequency-domain instruments. While CW systems can be built more inexpensively than time- or frequency-domain instruments, determination of tissue constituents via quantitative assessment of absorption and scattering properties is not possible. ${ }^{10}$ Instead, such systems can only be used to detect changes in hemoglobin concentration relative to baseline. ${ }^{14}$ However, estimates of the tissue absorption coefficient can be obtained by assuming reduced scattering coefficient from the literature values ${ }^{15}$ or by incorporating frequency-domain measurements to determine reduced scattering coefficient at selected wavelengths. ${ }^{16,17}$

Optical imaging of the breast can be accomplished with parallel plate geometry and slight compression of the breast, using an array of multiple fixed sources and detectors, ${ }^{18}$ or by scanning the source and detection fibers. ${ }^{19,20}$ Other approaches use ring or cup-like geometry to image the uncompressed breast. $^{14,17,21}$ Optical tomographic systems, including timedomain $^{21}$ and combined CW frequency-domain systems, ${ }^{17,18}$ can be used to obtain a three-dimensional (3-D) reconstruction of optical properties (and thus absolute chromophore concentrations) within the breast, whereas $\mathrm{CW}$ systems can be used to measure relative changes in chromophore concentrations. ${ }^{14}$

1083-3668/2017/\$25.00 @ 2017 SPIE 
However, tomographic systems are often costly, large in size, and require computationally demanding analysis for 3-D reconstruction.

Two-dimensional (2-D) maps of tissue optical properties can also be obtained from parallel plate geometry and scanning of source and detection fibers. ${ }^{10}$ These instruments typically use $\mathrm{CW}$ illumination and therefore cannot directly quantify absolute hemoglobin concentrations. Time-domain, parallel plate optical mammography systems exist; however, absorption coefficient maps were found to provide poor sensitivity to breast inhomogeneities. ${ }^{20}$ Another approach is a hybrid CW and frequency-domain system that operates in reflectance geometry and is equipped with a handheld probe moved in a grid pattern over the location of the tumor. ${ }^{16,22}$ This approach utilizes a fixed source-detector separation of $28 \mathrm{~mm}$ to probe an estimated mean depth of $10 \mathrm{~mm}$. This system has been used to quantify differences in optical properties between breast tumors and healthy tissue ${ }^{16}$ and for monitoring neoadjuvant chemotherapy. ${ }^{22,23}$

Other modalities have been developed to distinguish between healthy tissue and cancerous tissue based on the mechanical properties. Clinical breast exam (CBE) is one such method that relies on the detection of stiffness contrast between breast lesions and healthy tissue, but results of CBE are inherently subjective. In addition, comparison of a lesion's size and stiffness with that of past exams is also challenging. Multiple approaches have been developed to attempt to quantify differences detected by palpation. In mechanical or tactile imaging, an array of force sensors records stresses resulting from pressing a probe against the tissue, ${ }^{24-26}$ or mechanical indentation with a single force sensor may be performed at multiple locations over the location of the mass. ${ }^{27,28}$ In addition, shear wave elastography can be used to noninvasively estimate elastic modulus in vivo, based on the speed of propagation of shear waves introduced by an acoustic pressure wave. $^{29,30}$

Aside from directly measuring this mechanical contrast, there exists an opportunity for an optical breast lesion-monitoring device that takes advantage of stiffness contrast by leveraging compressing of tissue. Compression in x-ray mammography provides multiple benefits, including reduction of motion blur, decreased radiation dose, and the prevention of tissue overlap. ${ }^{31}$ For diffuse optics, depth sensitivity depends in-part on the imaging geometry. In reflectance mode diffuse optical imaging, depth sensitivity varies based on the source-detector separation, with larger separation sampling deeper regions of tissue. As depth sensitivity is limited, tissue compression has the potential to increase detection of breast masses by decreasing total tissue thickness. In addition, compression over the location of a stiff, palpable breast lesion has the potential to reduce the thickness of the superficial healthy tissue without significantly reducing the size of the lesion.

Here, we describe an approach for imaging palpable breast lesions that is based on spatial frequency-domain imaging (SFDI). ${ }^{32}$ Using structured illumination at multiple spatial frequencies, absorption and scattering properties of tissue are quantified, producing "maps" of absolute hemoglobin concentration over an area spanning several centimeters. The technique requires a projector and $C C D$ camera, with each pixel on the CCD sensor functioning as a detector. This enables high spatial resolution without any moving components and without a complex array of optical fibers. Such a system has the potential for incorporation into an inexpensive handheld imaging device,

which could even be sent home with the patient for frequent self-monitoring. Spatially modulated light has previously been used for tomographic reconstruction of heterogeneities in phantoms located $3 \mathrm{~mm}$ beneath the surface, ${ }^{33}$ but the limits of depth sensitivity for SFDI have not yet been conclusively determined. While SFDI remains primarily sensitive to superficial layers of tissue, we will demonstrate that this approach is applicable for deeper, but palpable, breast lesions. The relatively low absorption coefficient of the healthy breast and the flexibility of the tissue present a good opportunity for measurement with this technique. In particular, we envision that by compression of soft tissue, such as healthy breast tissue, stiff, palpable lesions can be imaged within the depth sensitivity of SFDI. This potential is demonstrated using breast tissue-mimicking soft phantoms, where mechanical compression is used to image highly absorbing, stiff inclusions.

\section{Methods}

SFDI is a quantitative imaging method to assess tissue optical properties. Details about SFDI can be found elsewhere ${ }^{32}$ and are described only briefly here. SFDI utilizes projections of spatially modulated light to extract tissue optical properties, specifically the absorption, $\mu_{\mathrm{a}}$, and reduced scattering, $\mu_{\mathrm{s}}^{\prime}$, coefficients, over a wide-field area. Our benchtop SFDI system uses a miniature laser projector (SHOWWX, MicroVision, Redmond, Washington) with center wavelength $\lambda=642 \mathrm{~nm}$ for illumination of tissue. Reflected images are captured by an 8-bit CCD camera (Chameleon, PointGrey, Richmond, British Columbia) using an exposure time of $16.45 \mathrm{~ms}$. To avoid specular reflection, crossed polarizers (LPNIRE100-B, Thorlabs, Newton, New Jersey) were employed. ${ }^{34,35}$ Working distance between the imaged object and the camera was $d=17 \mathrm{~cm}$, with an area of illumination of $8 \mathrm{~cm} \times 8 \mathrm{~cm}$. A schematic of the imaging system is seen in Fig. 1.

Sinusoidal patterns for illumination were generated in MATLAB $^{\circledR}$ (Mathworks Inc., Natick, Massachusetts) at five spatial frequencies $\left(f_{x}=0,0.04,0.06,0.08,0.1 \mathrm{~mm}^{-1}\right)$. Tabassum et al. have previously demonstrated that spatial frequencies of $0 \mathrm{~mm}^{-1}$ (direct current, DC) and $0.1 \mathrm{~mm}^{-1}$ are alone sufficient for separation of absorption and reduced scattering coefficients. ${ }^{36}$ The projector used exhibits a nonlinear intensity response $(\gamma)$, which was characterized by projecting DC illumination onto a homogeneous phantom at a series of pixel values, and recording the average intensity detected by the camera. The input-output curve was fit to

(a)

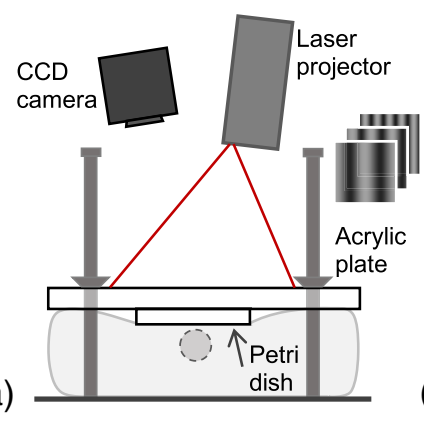

(b)

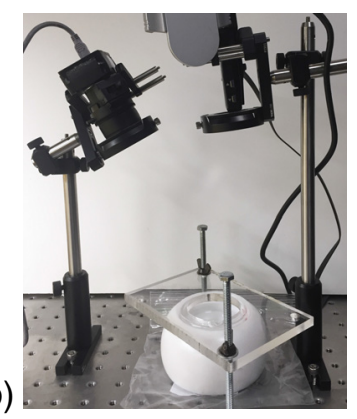

Fig. 1 (a) Schematic and (b) photo of SFDI system with phantom under compression. 
$V_{\text {out }}=A V_{\text {in }}^{\gamma}$

by applying a $\log$ transformation and using MATLAB's "polyfit" function with $n=1$ (linear fit). After determining the projector's characteristic $\gamma$ value, a correction was applied as illumination patterns were generated to compensate for this effect and ensure the projection of true sinusoidal patterns.

The projector operates by raster-scanning a laser across the projection area, with a refresh rate of $60 \mathrm{~Hz}$. Exposure time of the camera (16.45 ms) was chosen to capture one refresh of the laser projector, although it was not possible to synchronize the camera and projector precisely, resulting in artifacts. To eliminate these artifacts, images were acquired continuously at $15 \mathrm{fps}$, obtaining $\sim 12$ frames per illumination pattern. For each pixel, median value was calculated over these frames. Of each frequency, three evenly spaced phases (spatial shifts) were projected ( $0 \mathrm{deg}, 120 \mathrm{deg}$, and $240 \mathrm{deg}$ ). Five frequencies at three phases each were projected with a $0.1 \mathrm{~s}$ pause between each pattern. Total acquisition time was $\sim 20 \mathrm{~s}$.

Image analysis was performed according to the process described by Cuccia et al., ${ }^{32}$ which is summarized briefly here. For each frequency, the resulting three images are demodulated into one map of $\mathrm{AC}$ modulation $\left(M_{\mathrm{AC}}\right)$ according to

$M_{\mathrm{AC}}\left(x, f_{x}\right)=\frac{\sqrt{2}}{3} \sqrt{\left(I_{1}-I_{2}\right)^{2}+\left(I_{2}-I_{3}\right)^{2}+\left(I_{3}-I_{1}\right)^{2}}$,

where $I_{1}, I_{2}$, and $I_{3}$ are intensities for the three different phases, $f_{x}$ represents spatial frequency of illumination, and $x$ is the spatial location within the images. The same images are also obtained from a reference phantom of known optical properties. Cuccia et al. derived the following equation for diffuse reflectance, $R_{d}\left(f_{x}\right)$, of sinusoidal illumination that depends on optical properties of the sample $\left(\mu_{\mathrm{a}}\right.$ and $\left.\mu_{\mathrm{s}}^{\prime}\right)$ as well as spatial frequency of the illumination. ${ }^{32}$

$R_{d}\left(f_{x}\right)=\frac{3 A\left(\mu_{\mathrm{s}}^{\prime} / \mu_{\mathrm{tr}}\right)}{\left(\mu_{\mathrm{eff}}^{\prime} / \mu_{\mathrm{tr}}\right)\left(\mu_{\mathrm{eff}}^{\prime} / \mu_{\mathrm{tr}}\right)}$,

where $\mu_{\mathrm{tr}}=\mu_{\mathrm{a}}+\mu_{\mathrm{s}}^{\prime}$, and $\mu_{\mathrm{eff}}^{\prime}=\sqrt{3\left(\mu_{\mathrm{a}} \mu_{\mathrm{tr}}\right)+f_{x}^{2}} . A$ is a proportionality constant that incorporates refractive index of the medium. From Eq. (3), diffuse reflectance of the reference phantom with known optical properties may be calculated at each frequency, and diffuse reflectance of the sample is obtained multiplying this value by the ratio of sample and reference AC modulation. This division controls the absolute source intensity and modulation transfer function of the system. ${ }^{32}$
$R_{d, \text { samp }}\left(f_{x}\right)=\frac{M_{\mathrm{AC}, \mathrm{samp}}\left(f_{x}\right)}{M_{\mathrm{AC}, \mathrm{ref}}\left(f_{x}\right)} \cdot R_{d, \text { ref }}\left(f_{x}\right)$.

The result is a value of $R_{d}$ at multiple spatial frequencies for each pixel of the image. Values of $\mu_{\mathrm{a}}$ and $\mu_{\mathrm{s}}^{\prime}$ are then determined by fitting the data to Eq. (3) with the Levenberg-Marquardt algorithm (using MATLAB's "fsolve" function.)

Optical phantoms were created to mimic breast tissue in terms of stiffness as well as optical properties for healthy and cancerous tissues. Mechanical properties of healthy breast tissue have been reported previously to range between 3.25 and $22 \mathrm{kPa}$ for normal fat tissue and between 3.24 and $34 \mathrm{kPa}$ for normal glandular tissue. ${ }^{11,12}$ Breast cancer lesions are frequently stiffer than healthy tissue, with Young's modulus of 16 to $26 \mathrm{kPa}$ for ductal carcinoma in situ (DCIS) and 42 to $112 \mathrm{kPa}$ for invasive ductal carcinoma (IDC). ${ }^{11,12}$ In addition, Krouskop et al. found the stress-strain behavior of lesions to be more strongly nonlinear that that of a healthy breast. At higher strains (20\% precompression of tissue), the stiffness of both DCIS and IDC diverged greatly from that of healthy tissue. ${ }^{12}$ Optical properties of healthy breast tissue have been reported to be $\mu_{\mathrm{a}}=0.01$ to $0.05 \mathrm{~cm}^{-1}$ and $\mu_{\mathrm{s}}^{\prime}=10$ to $13 \mathrm{~cm}^{-1}$, for wavelengths of 600 to $700 \mathrm{~nm}$. The optical properties of breast cancer lesions are not usually reported on their own but as an absorption coefficient ratio between healthy and diseased tissues, ranging from 1.16 to 2.5 . $^{18,37,38}$

To mimic healthy breast tissue and palpable breast lesions, flexible polydimethyl siloxane (PDMS) (Ecoflex 00-10, Smooth-on, Macungie, Pennsylvania) phantoms were fabricated with stiffer breast cancer-mimicking inclusions embedded (SORTA-clear 40, Smooth-on, Macungie, Pennsylvania).

We fabricated optical phantoms using titanium dioxide $(0.3$ to $1.0 \mu \mathrm{m}$, Atlantic Equipment Engineers, Upper Saddle River, New Jersey) and Higgins black India ink (Chartpak, Inc., Leeds, Massachusetts) as absorption and scattering agents, respectively. The phantoms were molded in cylindrical containers, having a diameter of $8.2 \mathrm{~cm}$ and height of $6.4 \mathrm{~cm}$. Inclusions were spherical, with a diameter of $1.7 \mathrm{~cm}$. For the breast tumor-mimicking inclusions, varying amounts of ink were used to achieve a range of absorption coefficients, identified as types A, B, C, and D (Table 1).

For the background, we used $2400 \mathrm{~mL}$ each of Ecoflex parts $\mathrm{A}$ and $\mathrm{B}, 552 \mu \mathrm{L}$ of ink, and $8 \mathrm{~g}$ of $\mathrm{TiO}_{2}$. Absorption and scattering agents were combined with the highly viscous parts $A$ and $\mathrm{B}$ with the aid of an electric hand mixer, and both components were vacuum degassed separately for $\sim 1 \mathrm{~h} .1280 \mathrm{~mL}$ of silicone thinner (Smooth-on, Macungie, Pennsylvania) was added to part A to aid in mixing and degassing. The two components were then combined and mixed by hand. The final mixture was degassed for an additional $10 \mathrm{~min}$ after being transferred to the cylindrical molds.

Table 1 Optical and mechanical properties of phantoms. For optical properties of inclusions A-D, values in brackets indicate the contrast relative to the background optical properties: $\left(\mu_{\mathrm{a}, \text { inclusion }}-\mu_{\mathrm{a}, \text { background }}\right) / \mu_{\mathrm{a}, \text { background }}$ and $\left(\mu_{\mathrm{s} \text {, inclusion }}^{\prime}-\mu_{\mathrm{s}, \text { background }}^{\prime}\right) / \mu_{\mathrm{s}, \text { background }}^{\prime}$.

\begin{tabular}{lcccccc} 
& Background & Reference & Inclusion A & Inclusion B & Inclusion C & Inclusion D \\
\hline$\mu_{\mathrm{a}, 642}\left(\mathrm{~cm}^{-1}\right)$ & 0.033 & 0.052 & $0.058[0.8]$ & $0.079[1.4]$ & $0.091[1.7]$ & $0.104[2.1]$ \\
$\mu_{\mathrm{s}, 642}^{\prime}\left(\mathrm{cm}^{-1}\right)$ & 10.6 & 9.01 & $12.4[0.2]$ & $12.7[0.2]$ & $13.0[0.2]$ & $13.3[0.3]$ \\
Stiffness (OO scale) & 9.7 & 13.7 & 70.4 & 75.7 & 74.8 & 69.4 \\
\hline
\end{tabular}


For the inclusions, we used $1060 \mathrm{~mL}$ SORTA-clear part A, combined with $320 \mathrm{~mL}$ silicone thinner and $2.0 \mathrm{~g} \mathrm{TiO}_{2}$, mixed with an electric mixer and degassed for $\sim 1 \mathrm{~h}$. This mixture was divided evenly into four portions. To each, $26 \mathrm{~mL}$ of SORTAclear part B was added along with $69,94,117$, or $140 \mu \mathrm{L}$ of ink, mixed by hand, and transferred to spherical molds. The final mixtures were degassed for an additional $20 \mathrm{~min}$ in the molds, or until no more bubbles were visible.

Sixteen breast cancer-mimicking phantoms were created with each absorption contrast for the inclusion (A to D) at four different depths. Each phantom had one inclusion embedded in it at depths ranging from 13 to $22 \mathrm{~mm}$ (measured from surface of phantom to center of inclusion). Inclusion depth for each phantom is shown in Table 2.

The background of all 16 phantoms was made from one mixture of PDMS to ensure consistent optical properties of the background material. An additional homogeneous phantom with the same dimensions and mechanical properties was fabricated as a reference phantom for SFDI measurements. The homogeneous reference phantom was fabricated with a similar procedure to that described above, using $300 \mathrm{~mL}$ each parts $\mathrm{A}$ and $\mathrm{B}, 160$ $\mathrm{mL}$ thinner, $69 \mu \mathrm{L}$ ink, and $1.0 \mathrm{~g} \mathrm{TiO}_{2}$. For each type of inclusion (A to D), a larger homogeneous phantom was also made from the same batch of PDMS, for verification of optical properties. Optical properties of these phantoms were measured by a frequency-domain near-infrared spectroscopy (NIRS) system (OxiplexTS, ISS, Champaign, Illinois) at 690 and $830 \mathrm{~nm}$. Assuming a negligible wavelength dependence in absorption coefficient for India ink between 642 and $690 \mathrm{~nm}$, and a power law relationship in scattering coefficient, optical properties for $\lambda=642 \mathrm{~nm}$ were obtained and are summarized in Table 1 . The stiffness of the materials was also measured with a shore-OO durometer (Guangzhou Landtek Instruments, Guangzhou, China).

To evaluate the maximum depth of a detectable inclusion, as well as to quantify the contrast improvement by compression, we performed the following experiment for each phantom. A glass petri dish of diameter $6.3 \mathrm{~cm}$ was positioned over the center of the inclusion. A layer of mineral oil (Johnson \& Johnson, New Brunswick, New Jersey) was added between the glass and the phantom surface to guarantee uniform contact and eliminate entrapped air. An acrylic plate with a circular cutout was placed on top of the petri dish and used to apply evenly distributed force to the rim of the dish. The plate was lowered slightly to a standardized height to ensure good contact and to reduce effects from small height differences between the phantoms. Phantom height varied from 62.5 and $65.3 \mathrm{~mm}$, with a mean of $64.3 \mathrm{~mm}$. With percent compression defined as change

Table 2 Inclusion depth (measured from surface to center of inclusion) for all phantoms.

\begin{tabular}{lcccc} 
& $\begin{array}{c}\text { Depth 1 } \\
(\mathrm{mm})\end{array}$ & $\begin{array}{c}\text { Depth 2 } \\
(\mathrm{mm})\end{array}$ & $\begin{array}{c}\text { Depth 3 } \\
(\mathrm{mm})\end{array}$ & $\begin{array}{c}\text { Depth 4 } \\
(\mathrm{mm})\end{array}$ \\
\hline$\mu_{\mathrm{a}, \text { inclusion }}=0.058 \mathrm{~cm}^{-1}$ & 14.0 & 17.2 & 20.3 & 22.2 \\
$\mu_{\mathrm{a}, \text { inclusion }}=0.079 \mathrm{~cm}^{-1}$ & 14.4 & 16.3 & 20.0 & 22.2 \\
$\mu_{\mathrm{a}, \text { inclusion }}=0.091 \mathrm{~cm}^{-1}$ & 12.7 & 17.4 & 18.4 & 21.9 \\
$\mu_{\mathrm{a} \text {,inclusion }}=0.104 \mathrm{~cm}^{-1}$ & 13.0 & 17.1 & 19.0 & 22.1 \\
\hline
\end{tabular}

in phantom height divided by initial height, initial phantom compression was $\sim 3 \%$. The phantom was then further compressed in four increments: $16 \%, 31 \%, 47 \%$, and $55 \%$ compression. SFDI images were acquired at each compression level. The homogeneous reference phantom was imaged with the same compression levels to avoid height differences and ensure consistent illumination between the sample and reference phantom. Changes in intensity that occurred as the phantom was compressed could be ignored due to the nature of Eq. (4), which considers only a ratio between sample and reference intensity.

However, as the same sinusoidal illumination patterns were used for all compression levels, a nonnegligible decrease in spatial frequency occurred as the distance between sample and projector was increased. Effective spatial frequencies (corrected for divergence of projector) were determined and used in fitting optical properties. This divergence was quantified by imaging a calibration pattern of known size, which was placed on the surface of the phantom. Images were acquired at each compression level under ambient illumination, which were used to establish spacing (pixel/mm) for each level. The calibration pattern was then projected onto the phantom at each compression level. From these images, the width of the pattern in pixels was determined and converted to width in $\mathrm{mm}$. Due to this divergence, the spatial frequency of illumination at highest compression level was found to be $84 \%$ of that at lowest compression level. The change in spatial frequency was taken into account for further processing, which was based on applying Eqs. (2)-(4) for computing 2-D maps of $\mu_{\mathrm{a}}$ and $\mu_{\mathrm{s}}^{\prime}$.

In order to evaluate whether the optical properties of the embedded inclusions yield a contrast comparable to the literature values in humans using reflectance-based single-sourcedetector signals, we performed the following experiments. Using a frequency-domain NIRS system (OxiplexTS, ISS, Champaign, Illinois), one 400- $\mu$ m-diameter detection fiber and one 400- $\mu \mathrm{m}$-diameter source fiber (FT400EMT, Thorlabs, Newton, New Jersey) for $690 \mathrm{~nm}$ were positioned at a separation of $28 \mathrm{~mm}, \sim 2 \mathrm{~mm}$ from the surface of the phantom. The sourcedetector pair was then translated along the surface of the phantoms with a total of $40 \mathrm{~mm}$, with the inclusion located in the center of the scanned path. The direction of motion was perpendicular to the fiber probe direction and DC intensity was measured every $2.25 \mathrm{~mm}$. From these measurements, an estimate of change in absorption coefficient from baseline was obtained from the modified Beer-Lambert law ${ }^{39}$

$\Delta \mu_{\mathrm{a}}(x)=\frac{1}{r \cdot \mathrm{dpf}} \ln \left[\frac{\mathrm{DC}(0)}{\mathrm{DC}(x)}\right]$,

where $r$ is the source-detector separation, and dpf is the differential pathlength factor, estimated to be 10.7 (from sourcedetector separation and baseline $\mu_{\mathrm{a}}$ and $\mu_{\mathrm{s}}^{\prime}$ at $690 \mathrm{~nm}$, assuming semi-infinite medium). ${ }^{40} \mathrm{DC}(x)$ is the $\mathrm{DC}$ intensity measured at a given spatial location. $\mathrm{DC}(0)$ is the intensity value of the background. The result is a linescan representing $\Delta \mu_{\mathrm{a}, 690}$ at varying spatial locations over the center of the inclusion for each phantom.

\section{Results}

Dependent on the inclusion optical properties, the inclusion was detectable by SFDI at low levels of compression for some but not all phantoms. With few exceptions, the inclusion was undetectable without compression. At full compression 
(55\% change in height), the inclusion was visible in all cases. Representative 2-D maps of $\mu_{\mathrm{a}}$ for 8 of the 16 phantoms at two selected compression levels are shown in Fig. 2. To aid visual comparison, background $\mu_{\mathrm{a}}$ for each phantom has been standardized to the mean value.

For each phantom at maximum compression, the center of the inclusion was located by cropping the absorption coefficient map to include the known center location and exclude artifacts, then applying a Gaussian blur filter (MATLAB's "imguassfilt" function) of $\sigma=3$, and obtaining the location of maximum pixel value. Locations at all other compression levels were obtained by applying a known correction for the slight translation in the frame resulting from compression. An average cross section over five pixels, corresponding to a width of $2.3 \mathrm{~mm}$, was obtained for each absorption coefficient map (16 phantoms and 5 compression levels each). The average optical properties of the background (defined as the average values over a region $\sim 10$ to $12 \mathrm{~mm}$ away from the center of the inclusion on either side) did not vary significantly with the level of compression. For increasing levels of compression, mean background $\mu_{\mathrm{a}}$ was found to be $0.031 \pm 0.004,0.031 \pm 0.003,0.030 \pm 0.003$, $0.030 \pm 0.003$, and $0.030 \pm 0.003 \mathrm{~cm}^{-1}$, and mean background $\mu_{\mathrm{s}}^{\prime}$ was found to be $11.0 \pm 0.4,11.1 \pm 0.4,11.1 \pm 0.4$, $11.1 \pm 0.3$, and $11.1 \pm 0.3 \mathrm{~cm}^{-1}$, demonstrating that height changes were accurately accounted. Greater variation occurred among measurements. For four independent measurements, mean background $\mu_{\mathrm{a}}$ was found to be $0.034 \pm 0.002$, $0.032 \pm 0.002,0.027 \pm 0.002$, and $0.028 \pm 0.002 \mathrm{~cm}^{-1}$, and mean background $\mu_{\mathrm{s}}^{\prime}$ was found to be $10.7 \pm 0.2,11.0 \pm 0.2$, $11.3 \pm 0.1$, and $11.5 \pm 0.2 \mathrm{~cm}^{-1}$.

To further validate the quantification of optical properties by the SFDI system, eight phantoms were considered, which contained inclusions deeper than $18 \mathrm{~mm}$, as the inclusion was in all cases undetectable without compression. From four independent SFDI measurements of these eight uncompressed phantoms, mean optical properties of $\mu_{\mathrm{a}}=0.030 \mathrm{~cm}^{-1}$ and $\mu_{\mathrm{a}}=11.1 \mathrm{~cm}^{-1}$ were obtained. Compared with the values in Table 1 (measured with frequency-domain NIRS), this represents a $9.0 \%$ error in quantifying $\mu_{\mathrm{a}}$, and a $5.2 \%$ error in quantifying $\mu_{\mathrm{s}}^{\prime}$. However, this may in-part represent error in estimating optical properties at $642 \mathrm{~nm}$ using NIRS measurements at 690 and $830 \mathrm{~nm}$.
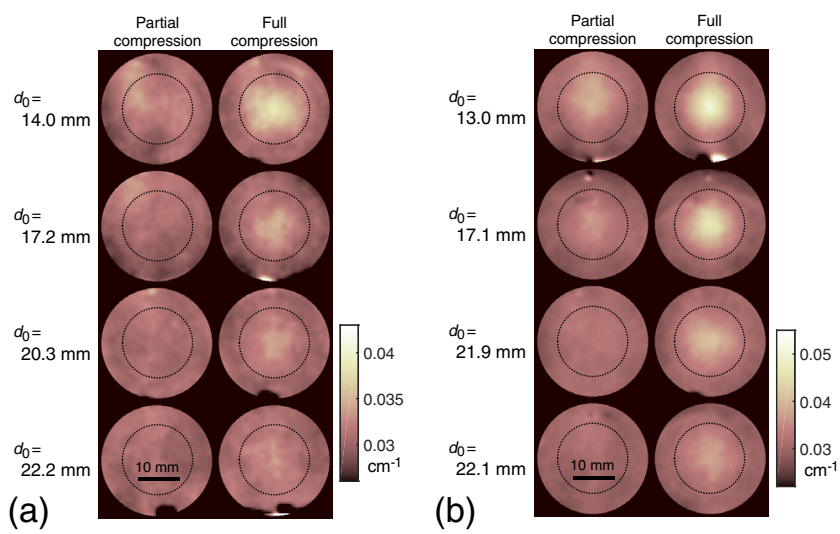

Fig. 2 Absorption coefficient maps for four phantoms containing inclusions of $\mu_{\mathrm{a} \text {,inclusion }}=0.058 \mathrm{~cm}^{-1}$ at (a) different initial depths and four phantoms containing inclusions of $\mu_{\mathrm{a} \text {,inclusion }}=0.104 \mathrm{~cm}^{-1}$ (b) at partial compression (31\%) and full compression (55\%). Dotted circle represents the size of the inclusion $(1.7 \mathrm{~cm})$.
For further analysis, the background optical properties were subtracted from each result. The SFDI measurements were repeated four times and average $\Delta \mu_{\mathrm{a}}$ cross sections are shown in Fig. 3. From each cross section, absorption contrast

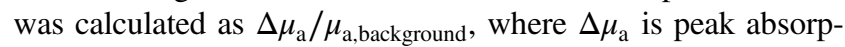
tion coefficient minus the baseline absorption coefficient for each measurement, and $\mu_{\text {a,background }}$ is $0.030 \mathrm{~cm}^{-1}$, the mean background absorption determined above.

Using the center locations identified above, reduced scattering coefficient cross sections were obtained with the same process and shown in Fig. 4. No significant increase in scattering is observed at the location of the inclusion, and compression does not have a significant effect on scattering cross sections. This is to be expected since scattering contrast was small, as seen in Table 1.

When the inclusion depth was increased for a given inclusion type (moving left to right across Figs. 3 and 4), optical

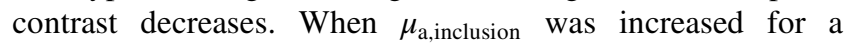
given inclusion depth (moving down a column in Figs. 3 and 4), the optical contrast increases. Since the inclusion effectively moves closer to the surface by means of compression, we estimated the effective inclusion depth during a given compression level by

(a)

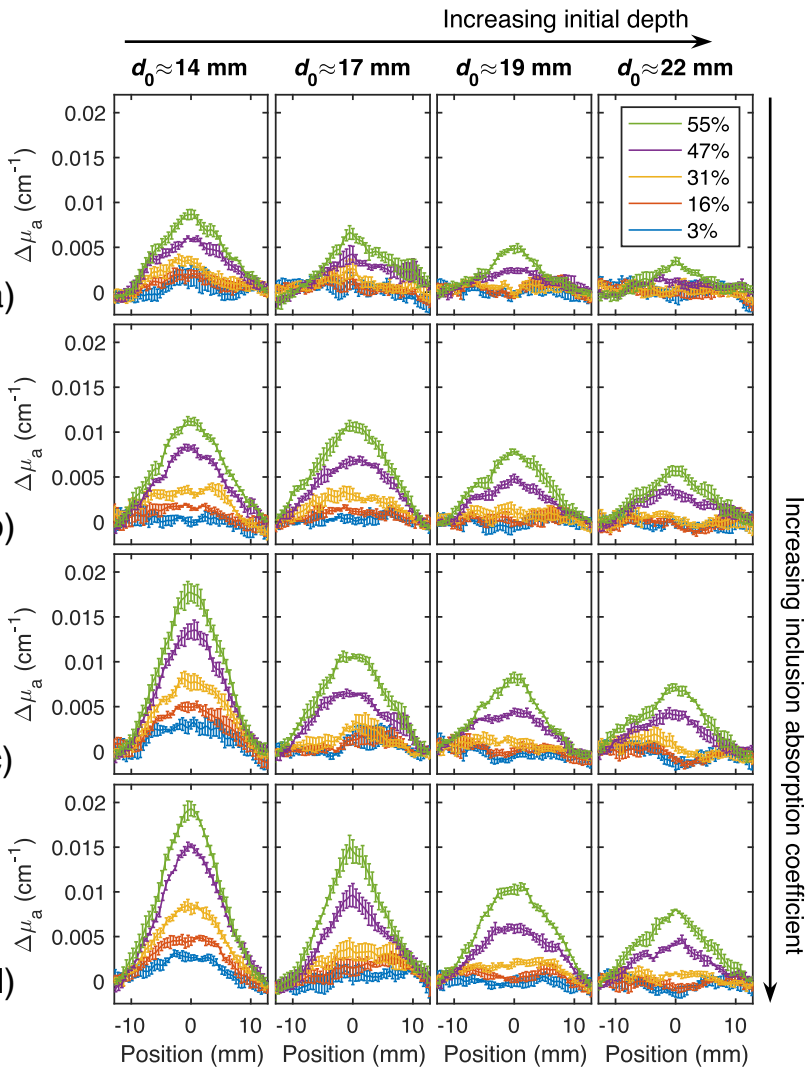

Fig. 3 Absorption coefficient cross sections for each phantom, at five levels of compression, percent compression defined as change in phantom height divided by initial height. (a) $\mu_{\text {a,inclusion }}=0.058 \mathrm{~cm}^{-1}$, (b) $\mu_{\mathrm{a} \text {,inclusion }}=0.079 \mathrm{~cm}^{-1}, \quad$ (c) $\mu_{\mathrm{a} \text {,inclusion }}=0.091 \mathrm{~cm}^{-1}$, and (d) $\mu_{\mathrm{a} \text {,inclusion }}=0.104 \mathrm{~cm}^{-1}$. The inclusion is positioned at the center of the cross section. As the absorption coefficient of the inclusion increases, the contrast measured with SFDI increases. Similarly, as the compression level increases, the effective inclusion depth decreases and the contrast increases as well. 


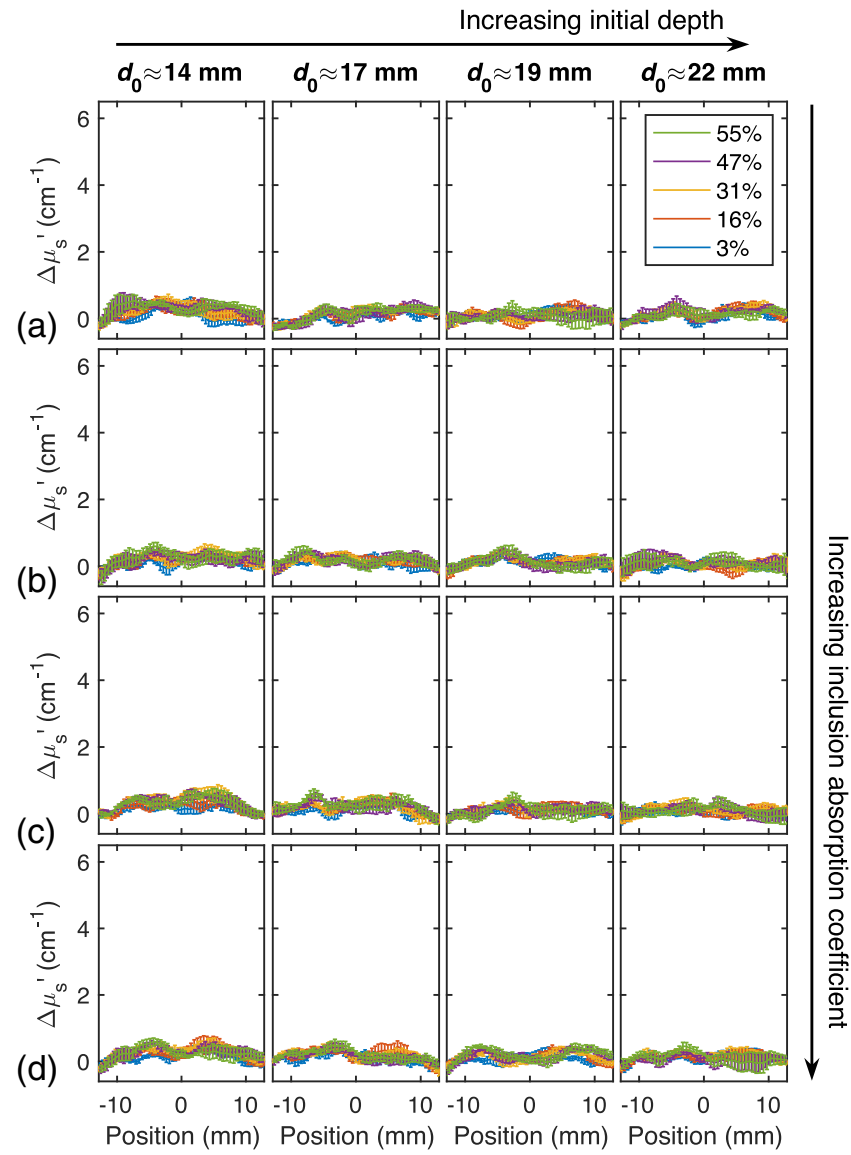

Fig. 4 (a)-(d) Reduced scattering coefficient cross sections for each phantom, at five levels of compression, percent compression defined as change in phantom height divided by initial height. No significant scattering contrast is detected, and no significant change with compression. The $y$-axis is scaled to correspond to the same percentage change as in Fig. 3.

$$
d=\left(\frac{h}{h_{0}}\right) \times d_{0},
$$

where $h_{0}$ and $h$ are the initial and compressed heights of the phantom, respectively, $d_{0}$ is the initial depth of the inclusion (measured from surface to center of the inclusion), and $d$ is the estimated final depth during compression.

Using the estimated depth, Fig. 5(a) shows the optical contrast versus estimated final depth for all phantoms and all compression levels. The optical contrast decreases exponentially with depth for all $\mu_{\mathrm{a} \text {,inclusion. Best fit lines are calculated }}$ with MATLAB's "fit" function, model type "exp1" (single-term exponential). For SFDI, equations of exponential best fit are as follows: contrast $=1.57 e^{-0.30 d}$, contrast $=$ $2.46 e^{-0.29 d}$, contrast $=2.69 e^{-0.29 d}$, contrast $=2.62 e^{-0.26 d}$, for $\mu_{\text {a,inclusion }}=0.058,0.079,0.091$, and $0.104 \mathrm{~cm}^{-1}$, respectively. Figure 5(b) shows the optical contrast from the single sourcedetector separation NIRS measurements, also decreasing exponentially with increase in inclusion depth. Optical contrast is calculated as peak $\Delta \mu_{\mathrm{a}} / \mu_{\text {a,background, where } \mu_{\text {a,background }}}$ is $0.033 \mathrm{~cm}^{-1}$, based on the NIRS measurements at $690 \mathrm{~nm}$. Equations of exponential best fit are contrast $=$ $1.46 e^{-0.14 d}, \quad$ contrast $=2.70 e^{-0.13 d}, \quad$ contrast $=6.77 e^{-0.19 d}$, contrast $=3.89 e^{-0.14 d}$ for $\mu_{\mathrm{a} \text {,inclusion }}=0.058,0.079,0.091$, and $0.104 \mathrm{~cm}^{-1}$, respectively. Figure 5(c) compares contrast measured with SFDI only at highest compression level to that measured with NIRS (no compression), showing that the contrast between the inclusion and background as measured with SFDI is higher or comparable to conventional NIRSbased measurements. The reported NIRS contrast is based on the modified Beer-Lambert law, which assumes that the scattering coefficient does not change. This assumption is justifiable based on Fig. 4, which demonstrates that the scattering contrast is negligible.
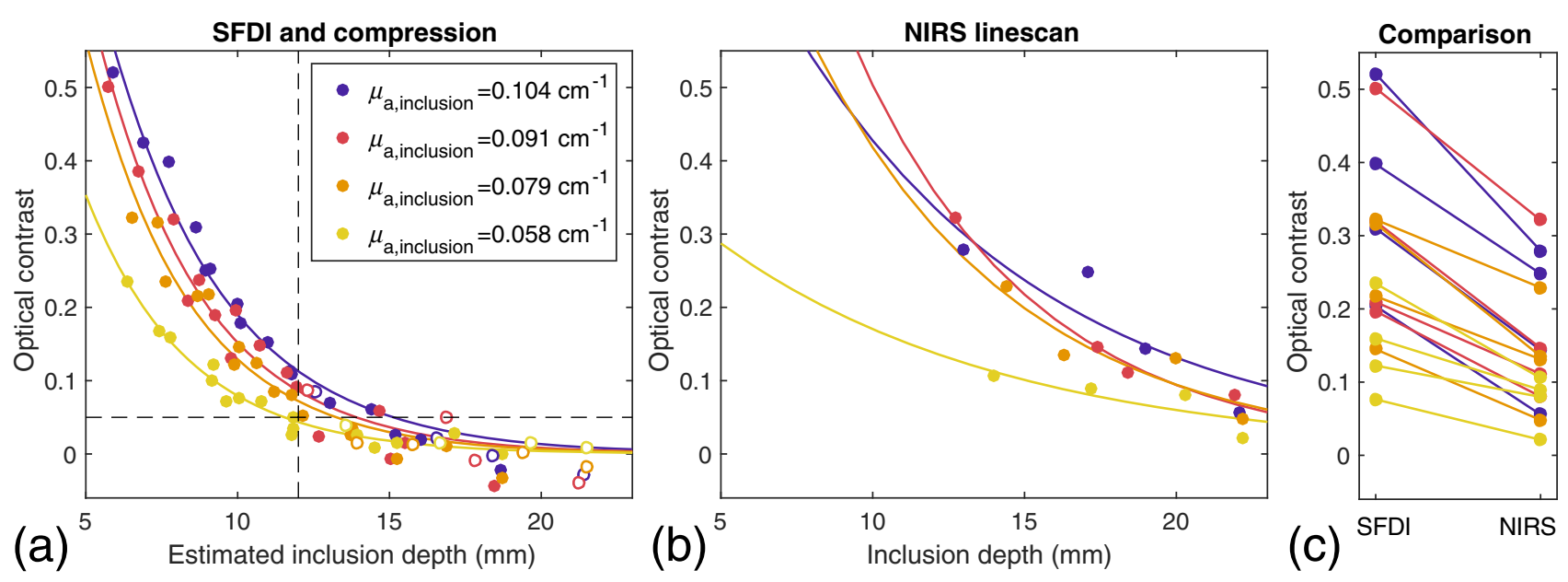

Fig. 5 Measured contrast decreases exponentially with depth for (a) SFDI at all compression levels and (b) single source-detector separation NIRS. Although depth during compression was not known precisely, the estimate used here shows the expected exponential relationship, with dependence on

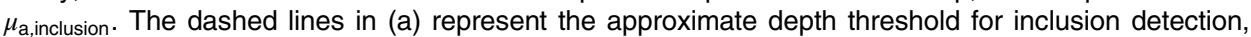
$12 \mathrm{~mm}$. At lower depths, contrast is greater than 0.05 for each of the four exponential fits. Open circles represent measurements of uncompressed phantoms. For each of the 16 phantoms, (c) compares optical contrast from SFDI at highest compression level to that measured with single source-detector separation NIRS, demonstrating that a similar level of contrast can be achieved by this method. 


\section{Discussion}

We have demonstrated that stiff, more highly absorbing inclusions embedded in a soft phantom can be imaged with SFDI even at initial depths of over $20 \mathrm{~mm}$. The principle of this finding is based on compression, where a stiff inclusion is effectively brought closer to the surface, making it possible to be imaged within the depth sensitivity of SFDI. The results of Fig. 5(a) indicate that the effect on measured contrast by compression can be described by a reduction in depth of the inclusion. Regardless of initial inclusion depth, compression to a final depth of less than $\sim 12 \mathrm{~mm}$ appears to be necessary to achieve reliable detection of the inclusion, defined as contrast above $5 \%$ [Fig. 5(a)]. SFDI with compression [Fig. 5(a)] showed similar contrast as the NIRS measurements [Fig. 5(b)].

We believe that this approach has potential for imaging palpable breast lesions in vivo in humans. For a demonstration of the approach, we have used optical phantoms for which the background material has $\mu_{\mathrm{a}}=0.033 \mathrm{~cm}^{-1}$ and $\mu_{\mathrm{s}}^{\prime}=10.6 \mathrm{~cm}^{-1}$ at $642 \mathrm{~nm}$, containing inclusions with $\mu_{\mathrm{a}}=0.058,0.079,0.091$, and $0.104 \mathrm{~cm}^{-1}$ and $\mu_{\mathrm{s}}^{\prime}=12.4,12.7,13.0$, and $13.3 \mathrm{~cm}^{-1}$. Future in vivo studies will be performed at higher wavelengths than what is used here, in order to maximize tissue penetration. Typical total hemoglobin concentration in healthy breast tissue is $\sim 20 \mu \mathrm{M},{ }^{10,13}$ which corresponds to $\mu_{\mathrm{a}}<0.04 \mathrm{~cm}^{-1}$ for wavelengths between 680 and $820 \mathrm{~nm}$ and to $\mu_{\mathrm{a}} \approx 0.026 \mathrm{~cm}^{-1}$ at $730 \mathrm{~nm}$. Thus, the absorption of the background material used was representative of the absorption of healthy breast tissue for wavelengths in the near-infrared range.

The four inclusion types used (A to D) had absorption contrasts of $0.8,1.4,1.8$, and 2.1 relative to that of the background. Using more conventional imaging systems with large sourcedetector distances, optical contrasts $\left(\Delta \mu_{\mathrm{a}} / \mu_{\text {a,background }}\right)$ have been reported in the literature, including $1.5,{ }^{37} 0.37,{ }^{38}$ and 0.16 . ${ }^{18}$ However, it is likely that the lower contrasts reported in the literature underestimate true tumor/normal contrast due to partial volume effects. For example, in one study using the University of California, Irvine's diffuse optical spectroscopy and imaging system, the authors reported a mean total hemoglobin concentration of $23.2 \mu \mathrm{M}$ in healthy breast, which increases to $35.1 \mu \mathrm{M}$ in breast tumors (contrast of 0.5). ${ }^{16}$ Even though the authors acknowledged the effect of tumor depth on these results, depth is not directly assessed, and the contrast reported may represent a significant underestimation of total hemoglobin in breast tumors. The contrasts reported here from single source-detector linescan measurements are comparable to breast tumor absorption contrasts found in the literature. Even in the case of the highest absorbing inclusion studied here $\left(\mu_{\mathrm{a}}=0.104,3.1\right.$ times higher than background), single source-detector linescan measurements yielded contrast of less than 0.5 even at the shallowest depth studied.

The initial depths of the inclusions (12.7 to $22.2 \mathrm{~mm}$, corresponding to 4.2 to $13.7 \mathrm{~mm}$ when measured from surface to edge of inclusion) were also comparable to that of palpable breast lesions. ${ }^{41}$ In a study of both lymph-node metastases in both palpable and nonpalpable breast tumors, node-negative tumors were located a median of $9.7 \mathrm{~mm}$ from the skin to edge of tumor and node-positive tumors a median of $7.2 \mathrm{~mm}$ from the skin. ${ }^{42}$ Another study of invasive cancer visible on preoperative ultrasound found $39 \%$ of patients to have a tumor located less than $3 \mathrm{~mm}$ from the skin. ${ }^{43}$ The depths studied here are applicable to most palpable breast lesions and possibly to a large portion of nonpalpable breast lesions as well.
The soft PDMS used here (Ecoflex 00-10) has been used to mimic the mechanical properties of breast tissue and found to have an elastic modulus of $11 \mathrm{kPa}$ at $1 \%$ strain. ${ }^{44}$ Samani et al. reported the elastic modulus of healthy breast tissue to be under $4 \mathrm{kPa},{ }^{11}$ whereas Krouskop et al. reported values up to $22 \mathrm{kPa},{ }^{12}$ indicating the material used here is comparable in stiffness to healthy breast tissue. By conversion from shore A hardness, the elastic modulus of SORTA-clear 40 is estimated to be $1.3 \mathrm{MPa},{ }^{45}$ significantly stiffer than that of breast malignancies $\left(\sim 50\right.$ to $\left.100 \mathrm{kPa}^{11,12}\right)$. Further experiments are needed with inclusions that more closely mimic the stiffness of breast tumors.

In the translation of this technique to in vivo breast imaging, blanching of tissue is a significant concern. Carp et al. have shown $6 \mathrm{lbs}$ of compressive force $(2.6 \mathrm{kPa}$ if contact area is assumed to be 16 in Ref. 2) sufficient to induce hemodynamic changes in the breast. ${ }^{46} \mathrm{~A}$ decrease in total hemoglobin concentration on the order of $10 \%$ was observed, demonstrating that a significant blood volume remains in the tissue at this level of compression. Krouskop et al. reported the stress-strain curve of fat tissue in the breast to remain roughly linear up to strains of $30 \%$ and reported an elastic modulus of $\sim 15 \mathrm{kPa}$ for the tissue. ${ }^{12}$ Thus, at $30 \%$ strain (breast compressed to $70 \%$ of starting thickness), the tissue experiences a stress of $\sim 4.5 \mathrm{kPa}$, or $34 \mathrm{mmHg}$. This pressure estimate is significantly lower than arterial blood pressure, thus, indicating the possibility of significant compression of the breast while maintaining hemoglobinbased absorption contrasts between lesion and healthy tissue.

In addition to monitoring of palpable breast lesions, we envision SFDI with compression also having potential for frequent monitoring of neoadjuvant chemotherapy. Using diffuse optical methods, multiple studies have found changes in total hemoglobin concentration or tissue oxygen saturation of hemoglobin that may be predictive of complete response to therapy, ${ }^{47-50}$ and an SFDI-based home usage device could allow for frequent monitoring to elucidate changes occurring in tumor vasculature in the time between chemotherapy doses. SFDI has been used previously to monitor chemotherapy in a mouse tumor mode ${ }^{36}$ and we envision translation to human monitoring. For the experiments presented here, time to acquire one absorption map was $\sim 20 \mathrm{~s}$; however, this time can be significantly reduced by the use of a higher frame rate camera, reduction of noise (eliminating the need to average multiple frames), and the selection of fewer spatial frequencies.

\section{Disclosures}

The authors have no relevant financial interests in this paper.

\section{References}

1. U.S. Cancer Statistics Working Group, "United States cancer statistics: 1999-2014 incidence and mortality web-based report," U.S. Department of Health and Human Services, Centers for Disease Control and Prevention and National Cancer Institute, Atlanta (2017), www.cdc. gov/uscs (9 August 2017).

2. "Breast cancer screening and diagnosis (version 2.2016)," Nation Comprehensive Cancer Network, 2016, https:/www.nccn.org/ professionals/physician_gls/pdf/breast-screening.pdf (1 January 2017).

3. J. A. Harvey et al., "Short-term follow-up of palpable breast lesions with benign imaging features: evaluation of 375 lesions in 320 women," Am. J. Roentgenol. 193(6), 1723-1730 (2009).

4. C. S. Giess et al., "Risk of malignancy in palpable solid breast masses considered probably benign or low suspicion: implications for management," J. Ultrasound Med. 31(12), 1943-1949 (2012). 
5. O. Graf et al., "Follow-up of palpable circumscribed noncalcified solid breast masses at mammography and US: can biopsy be averted?" Radiology 233(3), 850-856 (2004).

6. Y.-M. Park et al., "Palpable breast masses with probably benign morphology at sonography: can biopsy be deferred?" Acta Radiol. 49(10), 1104-1111 (2008).

7. R. Choe and T. Durduran, "Diffuse optical monitoring of the neoadjuvant breast cancer therapy," IEEE J. Sel. Top. Quantum Electron. 18(4), 1367-1386 (2012).

8. C. Fiorentino et al., "Accuracy of mammography and echography versus clinical palpation in the assessment of response to primary chemotherapy in breast cancer patients with operable disease," Breast Cancer Res. Treat. 69(2), 143-151 (2001).

9. K. H. Koga et al., "Monitoring the response to neoadjuvant chemotherapy in breast cancer," in Neoadjuvant Chemotherapy-Increasing Relevance in Cancer Management, pp. 25-48, InTech, Rijeka, Croatia (2013).

10. D. Grosenick et al., "Review of optical breast imaging and spectroscopy," J. Biomed. Opt. 21(9), 091311 (2016).

11. A. Samani, J. Zubovits, and D. Plewes, "Elastic moduli of normal and pathological human breast tissues: an inversion-technique-based investigation of 169 samples," Phys. Med. Biol. 52, 1565-1576 (2007).

12. T. Krouskop et al., "Elastic moduli of breast and prostate tissue under compression," Ultrason. Imaging 20(4), 260-274 (1998).

13. D. R. Leff et al., "Diffuse optical imaging of the healthy and diseased breast: a systematic review," Breast Cancer Res. Treat. 108(1), 9-22 (2008).

14. M. L. Flexman et al., "Optical biomarkers for breast cancer derived from dynamic diffuse optical tomography," J. Biomed. Opt. 18, 096012 (2013).

15. P. G. Anderson et al., "Broadband optical mammography: chromophore concentration and hemoglobin saturation contrast in breast cancer," PLoS One 10(3), e0117322 (2015).

16. A. Leproux et al., "Assessing tumor contrast in radiographically dense breast tissue using diffuse optical spectroscopic imaging (DOSI)," Breast Cancer Res. 15(5), R89 (2013).

17. J. Wang et al., "In vivo quantitative imaging of normal and cancerous breast tissue using broadband diffuse optical tomography," Med. Phys. 37(7), 3715-3724 (2010).

18. R. Choe et al., "Differentiation of benign and malignant breast tumors by in-vivo three-dimensional parallel-plate diffuse optical tomography," J. Biomed. Opt. 14(2), 024020 (2009).

19. D. Grosenick et al., "Time-domain scanning optical mammography: I. Recording and assessment of mammograms of 154 patients," Phys. Med. Biol. 50(11), 2429-2449 (2005).

20. P. Taroni et al., "Time-resolved optical mammography between 637 and $985 \mathrm{~nm}$ : clinical study on the detection and identification of breast lesions," Phys. Med. Biol. 50(11), 2469-2488 (2005).

21. T. Yates et al., "Optical tomography of the breast using a multi-channel time-resolved imager," Phys. Med. Biol. 50(11), 2503-2517 (2005).

22. S. Ueda et al., "Baseline tumor oxygen saturation correlates with a pathologic complete response in breast cancer patients undergoing neoadjuvant chemotherapy," Cancer Res. 72(17), 4318-4328 (2012).

23. A. E. Cerussi et al., "Predicting response to breast cancer neoadjuvant chemotherapy using diffuse optical spectroscopy," Proc. Natl. Acad. Sci. U. S. A. 104(10), 4014-4019 (2007).

24. C. Van Nguyen and R. F. Saraf, "Tactile imaging of an imbedded palpable structure for breast cancer screening," ACS Appl. Mater. Interfaces 6(18), 16368-16374 (2014).

25. V. Egorov et al., "Differentiation of benign and malignant breast lesions by mechanical imaging," Breast Cancer Res. Treat. 118(1), 67-80 (2009).

26. P. S. Wellman et al., "Tactile imaging of breast masses: first clinical report," Arch. Surg. 136(2), 204-208 (2001).

27. L. Sallaway et al., "Detecting solid masses in phantom breast using mechanical indentation," Exp. Mech. 54(6), 935-942 (2014).

28. S. M. Hosseini et al., "A medical tactile sensing instrument for detecting embedded objects, with specific application for breast examination," Int. J. Med. Rob. Comput. Assisted Surg. 6(1), 73-82 (2010).

29. M. A. B. Blank and J. F. Antaki, "Breast lesion elastography region of interest selection and quantitative heterogeneity: a systematic review and meta-analysis," Ultrasound Med. Biol. 43(2), 387-397 (2017).
30. D. O. Cosgrove et al., "Shear wave elastography for breast masses is highly reproducible," Eur. Radiol. 22(5), 1023-1032 (2012).

31. W. Branderhorst et al., "Mammographic compression-a need for mechanical standardization," Eur. J. Radiol. 84(4), 596-602, (2015).

32. D. J. Cuccia et al., "Quantitation and mapping of tissue optical properties using modulated imaging," J. Biomed. Opt. 14(2), 024012 (2009).

33. S. D. Konecky et al., "Quantitative optical tomography of sub-surface heterogeneities using spatially modulated structured light," Opt. Express 17(17), 14780-14790 (2009).

34. S. G. Demos and R. R. Alfano, "Optical polarization imaging," Appl. Opt. 36(1), 150-155 (1997).

35. S. L. Jacques, J. R. Roman, and K. Lee, "Imaging superficial tissues with polarized light," Lasers Surg. Med. 26(2), 119-129 (2000).

36. S. Tabassum et al., "Feasibility of spatial frequency domain imaging (SFDI) for optically characterizing a preclinical oncology model," Biomed. Opt. Express 7(10), 4154-4170 (2016).

37. D. Grosenick et al., "Time-domain scanning optical mammography: II. Optical properties and tissue parameters of 87 carcinomas," Phys. Med. Biol. 50(11), 2451-2468 (2005).

38. Q. Fang et al., "Combined optical and x-ray tomosynthesis breast imaging," Radiology 258(1), 89-97 (2011).

39. S. Fantini et al., "Non-invasive optical monitoring of the newborn piglet brain using continuous-wave and frequency-domain spectroscopy," Phys. Med. Biol. 44(6), 1543-1563 (1999).

40. M. Bhatt, K. R. Ayyalasomayajula, and P. K. Yalavarthy, "Generalized Beer-Lambert model for near-infrared light propagation in thick biological tissues," J. Biomed. Opt. 21(7), 076012 (2016).

41. W. T. Yang and C. Metreweli, "Assessment of factors affecting palpability of breast masses using ultrasonography in Chinese women," J. Ultrasound Med. 15(12), 807-812 (1996).

42. B. Ansari et al., "Distance of breast cancer from the skin and nipple impacts axillary nodal metastases," Ann. Surg. Oncol. 18(11), 3174-3180 (2011).

43. Y. H. Eom et al., "The distance between breast cancer and the skin is associated with axillary nodal metastasis," J. Surg. Oncol. 111(7), 824-828 (2015).

44. M. Ayyildiz, "A novel opto-electro-mechanical tactile sensor for breast cancer imaging," Koç University (2011).

45. Y. Menguc et al., "Wearable soft sensing suit for human gait measurement," Int. J. Rob. Res. 33(14), 1748-1764 (2014).

46. S. A. Carp et al., "Dynamic functional and mechanical response of breast tissue to compression," Opt. Express 16(20), 16064-16078 (2008).

47. Q. Zhu et al., "Breast cancer: assessing response to neoadjuvant chemotherapy by using US-guided near-infrared tomography," Radiology 266(2), 433-442 (2013).

48. H. Soliman et al., "Functional imaging using diffuse optical spectroscopy of neoadjuvant chemotherapy response in women with locally advanced breast cancer," Clin. Cancer Res. 16(9), 2605-2614 (2010).

49. M. G. Pakalniskis et al., "Tumor angiogenesis change estimated by using diffuse optical spectroscopic tomography: demonstrated correlation in women undergoing neoadjuvant chemotherapy for invasive breast cancer?" Radiology 259(2), 365-374 (2011).

50. A. E. Cerussi et al., "Diffuse optical spectroscopic imaging correlates with final pathological response in breast cancer neoadjuvant chemotherapy," Philos. Trans. A Math. Phys. Eng. Sci. 369(1955), 45124530 (2011).

Constance M. Robbins is a $\mathrm{PhD}$ student in Carnegie Mellon University's Department of Biomedical Engineering in the Biophotonics Lab led by Prof. Jana Kainerstorfer. She received her bachelor's degree in biomedical engineering from the University of Pittsburgh and began graduate study at Carnegie Mellon in 2015. Her research interests focus on optical methods for diagnosis and monitoring of breast cancer, including spatial frequency-domain imaging and dynamic near-infrared spectroscopy.

Guruprasad Raghavan is a research assistant at Carnegie Mellon University's Department of Biomedical Engineering in the Biophotonics Lab led by Prof. Jana Kainerstorfer. He received his MS degree in biomedical engineering from Carnegie Mellon University and is headed to Caltech for his $\mathrm{PhD}$. His research interests include optical monitoring of breast cancers and improving 
the signal processing workflow to extract functional information from conventional optical coherence tomography.

James F. Antaki is a professor of biomedical engineering at Carnegie Mellon University, with adjunct appointments in the Departments of Surgery and Bioengineering at the University of Pittsburgh. His research emphasizes development of circulatory support systems for children, feedback-control algorithms and decision-support for optimizing cardiac recovery, and a blood purification system for treating malaria. $\mathrm{He}$ is also developing methods to heighten the involvement of physicians in process of innovation and design of new medical devices.

Jana M. Kainerstorfer is an assistant professor of biomedical engineering at Carnegie Mellon University. Her research includes noninvasive optical imaging of tissue for disease detection and/or treatment monitoring, with a particular emphasis on diffuse optical spectroscopic imaging (with applications to the brain, breast, skin, and muscle). Her research spans from instrument development to innovative data analysis tools all the way to clinical applications. 